\title{
Automated Bidding in English Auctions Using Cognitive Agents in Mobile e-Commerce
}

\author{
Nandini Sidnal and S. S. Manvi
}

\begin{abstract}
There is an increasing demand to monitor and bid concurrently in multiple English auctions in order to procure the best deal for the desired products. This paper proposes a scheme to incorporate human intelligence and perspective (cognitive) in agents for bidding in multiple concurrent English auctions in mobile E-commerce. The bidder mobile agent is embedded with intelligence to take decisions on behalf of a bidder with regard to either continue bidding or withdraw from auctions. The agent ensures that the bidder receives an appropriate number of desired products from one of the participated relevant auction site that has better chances of winning with satisfaction.

Dynamic E-market and mobile bidder scenarios are simulated to train the cognitive agents for enabling them to take decisions on behalf of the bidder in real time. The proposed scheme performs better than the existing bidding strategy (based on limited E-market parameters) in terms of best possible bid value, bidder satisfaction, and budget.
\end{abstract}

Index Terms-BDI agent, mobile e-Commerce, mobile bidders.

\section{INTRODUCTION}

To bid in multiple online auctions simultaneously using handheld devices the bidder needs to fetch the required information from all the relevant auction sites and process it to compute the bid value. Since these operations have to be performed in a highly dynamic, uncertain and unpredictable environment it is important that the bidding process be automated. To implement automation, auction service providers and consumers can naturally be viewed as software agents since these are exactly the properties and types of environment that lend themselves to an agent-based approach as given in [1]. This paper proposes a scheme to incorporate human intelligence and human perspective (cognitive) in agents [2] for bidding in multiple concurrent English auctions in mobile E-commerce. Some of relevant works in regards to the bidding strategies for on-line English auctions include the following. An automated and data-driven bidding strategy is presented in [3]. A bidding strategy for obtaining goods in multiple overlapping English auctions is discussed in [4]. The work given in [5] discusses the design of an autonomous agent that can alleviate some of the problems like getting better deals and selecting a better auction by participating across multiple on-line auctions. This work is used for comparison with the proposed work. Design of algorithms for agents to use when participating in multiple

Manuscript received March 26, 2012; revised April 27, 2012.

N. S. Sidnal is with the Department of Computer Science and Engineering, KLESCET, Belgaum (e-mail sidnal.nandini@gmail.com)

S. S. Manvi is with Department of Electronics and Communication Engineering, Reva ITM, Bangalore. simultaneous English auctions, aiming to purchase multiple goods is explained in [6]. The authors in [7] investigate utility by maximizing bidding heuristics for agents that participate in multiple heterogeneous auctions. A heuristic bidding strategy for buying multiple goods in multiple English auctions is presented in [8]. Novel bidding algorithm that a software agent can use to obtain multiple goods from multiple overlapping English auctions is presented. The work in [9] presents a decision theoretic framework that an autonomous agent can use to bid effectively across multiple, simultaneous auctions. An approach to develop bidding agents that participate in multiple alternative auctions, with the goal of obtaining an item with a given probability is demonstrated in [10]. The authors in [11] develop a bidding agent for multiple heterogeneous auctions and build a heuristic decision making framework that an autonomous agent can exploit to tackle the problem of bidding across multiple auctions with varying start and end times and with varying protocols. Some of the drawbacks of existing bidding strategies are as follows: lack of intelligence in bidding, scalability for mobile bidders and efficient automation.

\section{PROPOSED WORK}

This section provides description of the proposed work in terms of computational models and agencies.

\section{A. Our Contributions}

Our contributions in this paper are as follows:

1) Cognitive architecture for software agents is adopted since it incorporates human intelligence and human perspective to compute the bid values, payoffs and to make decisions to withdraw or bid.

2) Autonomy is achieved in bidding in multiple sites simultaneously.

3) Bidders are ensured that they end up winning in only one of the relevant auction sites.

4) Bidders win in auctions that have relatively better payoff.

5) The co-bidders are assumed to be either risk neutral or risk averse.

6) The scheme is validated by comparing the simulation results obtained by our proposed scheme with the bidding strategies given in [12].

\section{B. Bidder Environment}

The mobile bidders register themselves using a handheld device in the registration desk of the regional gateway to participate in bidding. A light weight agent platform (LEAP) that hosts an agency to carry out the auctions and communicate with the bidders may be loaded on the 
handheld devices of the bidders. The agencies assist the bidder/s in fetching the set of relevant auctions based on their individual requirements and bids in all of them simultaneously. The agencies also assist in predicting the closing price of the relevant auctions that is used to calculate the bid increments so as to increase the probability of winning. The bidder needs to log-on to WWW only to initiate the bidding transactions and can get disconnected. Later the bidder can login to fetch the results. The bidder has to allot a budget and provide the specifications of the required products to the agency. The agency may interact with the bidder when required; else it autonomously participates in bidding following the defined norms of the bidder and the auctioneer. The participating bidders may be risk neutral, averse or proclave based on their aggressiveness to posses the product. The bidder may bid for multiple items from the same auction or from multiple auctions. A bidder can participate simultaneously in more than one auction to bid for either a single/multiple product and can bid, withdraw or reenter bidding at any time before the auction closes.

\section{Computational Models}

This section describes the computations required to compute the bid increment for placing the bid value, withdrawal conditions and utility value for a relevant auction. The bid increment value and in turn the bid value at $t i+1$ from the allotted budget amount $b$ is computed as given in equation 1. The budget is distributed equally to compute uniform and variable increments. bid value $(t i+1)=\operatorname{Max}+\left(b_{-} x\right) / n+D$ incr --- (1) where Max is current maximum bid value for the considered auction and $x$ is given by equation 2 and $n$ is total number of times the bidder is going to call in the relevant auction. $\mathrm{D}$ incr is given by equation 3. $x=1-\mathrm{Nbm} / \mathrm{Nb}$--- (2) where $\mathrm{Nbm}$ is number of times bid value is the maximum bid and $N b$ is number of calls. D_incr $=\left(\right.$ Max_bid value $\left.\left(t_{i}\right)\right) *$ (budget+risk+pcp) --- (3) Budget, risk and predicted closing price (pcp) factors are computations are given by equations (4), (5) and (6) respectively. budget $=\left(b \_\right.$bid value $\left.\left(t_{i}\right)\right) / b$--(4) where $b$ is the alloted budget to participate in an auction, bid value $\left(t_{i}\right)$ is the current bid value of the bidder. risk $=y / n 2$ --- (5) where $y$ is the ratio of number of risk bidders to total number of bidders in the considered relevant auctions and $n 2$ is number of concurrent relevant auctions. Risk bidders are the co-bidders whose bid value is greater than the bid value $\left(t_{i}\right) . p c p$ f actor $=\left(p c p(j) / a v g \_p c p\right){ }_{-} y$--- (6) where $p c p(j)$ is the predicted closing price of the considered $j^{\text {th }}$ relevant auction, $a v g \_p c p$ is the average of predicted closing prices of all concurrent relevant auctions.

The following are the conditions to withdraw from the relevant auction sites. If either of the condition is satisfied the bidder has to withdraw from that respective relevant auction. (1) If the increment value is greater than the remaining budget. (2) If the sum of elapsed time since the beginning of the auction and the anticipated time required for the auction to close is greater than the bidder's available time. (3) If the number of bids placed so far are greater than seventy percent of the total number of times the bidder is going to bid and if the number of times the bidder has bid the maximum value is lesser than twenty percent of the number of bids placed so far, then the bidder has to withdraw. (4) If difference between bid value and max bid value is greater than the bidder increment value. The utility value $U j$ of each relevant site at time $t_{i}$ is the remaining budget for each of the relevant auction sites at time ti.

\section{Agency}

In this section we discuss the gateway agency based on BDI model located in regional gateway.

1) Gateway Agency

The main task of this agency is to compute the bid value for English auctions. This process involves in two steps: 1) belief set generation for each relevant site based on bidder desires, and 2) Finalizing the intentions as bid, sleep or withdraw based on belief sets. Service directory identification and building is done using the scheme given in [13]. Case base reasoning [14] is used to identify relevant auctions for participation in bidding. The closing price prediction method [15] is used to predict the closing price before placing the bid value. 1) BDI agent: Figure 1 depicts the architecture of the bidder agent. It consists of bidders desires (products names and specifications), belief sets of each relevant site and intentions to achieve desires.

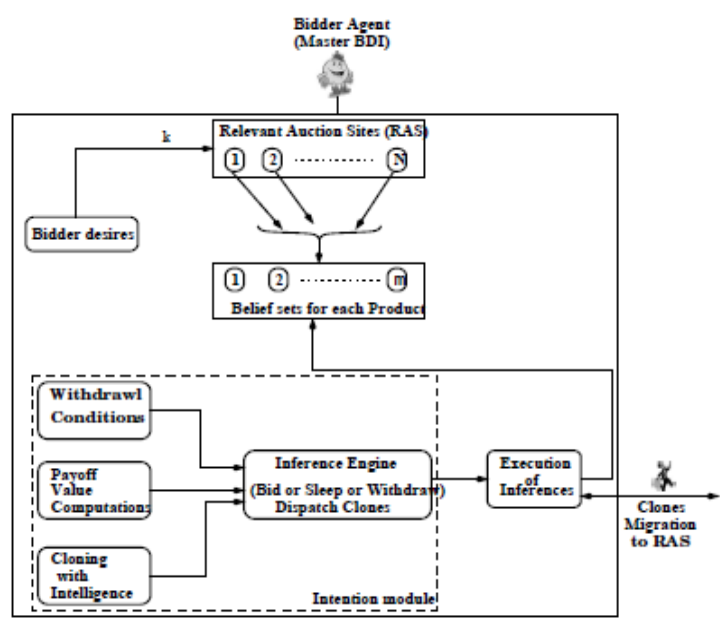

Fig. 1. BDI based bidder agent

In brief, the BDI functionality of the agency is as follows. Based on the bidder desires, belief sets are developed consisting of relevant auction information such as current max bid value, number of bidders, start time etc. The intention module consists of module to create clones, compute payoff, withdrawal conditions and an inference engine. The clones are created with intelligence of computing and placing bid value and are dispatched to the respective relevant auction sites to participate in bidding. The withdrawal conditions given in computational model helps to decide to withdraw bidding from an auction. Pay off is computed as given in computational model and is used to identify the relevant site that has better returns towards the end of participation. The inference engine identifies appropriate intention to be executed and decides whether to continue to bid, sleep or withdraw from participation and triggers the clones to act accordingly. This continues either till the bidder agent wins or withdraws from the relevant auctions. A clone is dispatched to each relevant auction to be stationed in the auction server either till the master wins the auction or withdraws from it. The clones initially communicate the history of the bidding information of the 
past auctions with the visited site for the product mentioned. This is based on bidder's desires and the opening bid of the current auction. As the auction progresses the clones compute the bid value using the computations (given in computational model) carried by them to the auction server and then communicate the bid value along with other required information such as the bid values of co bidders, current max-bid, number of co-bidders to the BDI master agent to decide on to bid, sleep or withdraw.

\section{E. Advantages and Limitations of the Proposed Scheme} Advantages

- The BDI agent participates on behalf of the bidder in multiple simultaneous auctions and is trained to bid in a highly dynamic, uncertain and unpredictable environment.

- The bidder's participation in the auctions can be ubiquitous.

- The connectivity of the bidder with WWW is required only to start the bidding transactions and can get disconnected until the results are obtained due to which the participation in the auctions is carried out in an uninterrupted manner regardless of spotty or no connectivity.

- The dynamic factors involved in E-auction such as behavior of risk averse/neutral bidders (fluctuations in bid rate of the co-bidders), remaining budget etc. have been considered to compute the bid value at each call.

- The BDI agent computes and bids concurrently in real time in all the participated relevant auctions.

- The bid increment computation does not require historical data.

- The BDI agent has the capability to withdraw its participation from the auctions when the payoff of the auction reduces.

Limitations

- The scheme does not take into account the discounts offered. This may be generalized.

- The BDI agent values all goods equally. This should be generalized to allow the agent to receive a demand curve from its user.

- The scheme is only designed for English auctions and needs to be extended to generalize to cover other forms of auction, such as Dutch, Vickrey and Double auctions.

- The list of relevant sites is to be updated till the bidder wins or withdraws

\section{Simulation MODEL}

The proposed frame work has been simulated in various network scenarios. It consists of Nc clusters, each consisting of $\mathrm{Nk}$ servers hosting $\mathrm{Nu}$ auctions in the fixed network, $\mathrm{Rg}$ regional gateways hosting $\mathrm{Nm}$ service directories and $\mathrm{Np}$
BDI agents to bid on behalf of bidders. Ns servers subset of $\mathrm{Nk}$ are connected to the gateway within the region. The simulated virtual market place consists of Naa number of active auctions offering $\mathrm{S}$ auction services in $\mathrm{Nk}$ auction servers. Nra a subset of Naa are relevant concurrent auctions auctioning the same product in a considered time window. Each auction auctions Pk products. The bidder participates in all the relevant auctions (Nra) concurrently. Each bidder is allotted a budget of RsBu. All the relevant auctions fix the opening bid value $O B$.

\section{A. Simulation Parameters and Procedure}

To illustrate some results of the simulation, following data is considered: $N c=1$ to $4, N k=1$ to $8, N u=50$ to $80, R g=1$, $N m=1, N p=2$ to $30, N s=1$ to $4, N a a=5$ to $500, N r a=2$ to $16, P k=10$ to $400, B u=25000$ to $35000, O B=200$ to 700 . The simulation procedure is as follows. (1) Create ' $n$ ' clones and allot budget to bid in the relevant auction sites. (2) Fill in bidding strategies in the clones and dispatch. (3) Master BDI agent will monitor bidding and decides weather to bid, sleep or withdraw from the site either till the master wins or with draws from the auction. Some of the performance measures considered in simulation is as follows. (1) Decision making The BDI is trained to compute bid value and bid, to sleep or to withdraw appropriately. (2) Best possible winning bid (bid value): The scheme ensures that the BDI agent will get the best deal for the desired product. (3) Bidders satisfaction: The bidder always wins the desired product with satisfaction. (4) Probability of winning in the relevant auction sites. (5) Budget allocation - budget is allotted based on predicted closing price and is re-allotted if required. (6) Agent Overhead - it is the additional code, data and state of the agent that utilizes the communication channel while the clones migrate to the servers and communicate with the master agent till the auction is won. (7) Computational overhead - it is the additional number of instructions executed to compute the bid value and the utility before placing the bid.

\section{RESUlts}

In this section, the results obtained with proposed work are discussed. The simulation is carried on core 2 duo machine using ' $C$ ' language. The analysis of the performance parameters are given in this section. The BDI agent (master) is trained to participate in concurrent relevant auction sites and later to decide whether to participate or withdraw from the auction for more than fifty scenarios of the bidding process. Few of them are presented in this paper.

Concurrent Participation: Figure 2 shows the concurrent participation of the clones of BDI agent in the relevant auction sites as they progress. The clones continue to participate in the auctions till they are withdrawn or retracted. Two of the clones withdraw early when they sensed that the rate of bidding of co-bidders is high and hence the payoff was very less. The clones in auction sites 4,5 and 6 withdraw when the payoff reduced relatively and/or the auctions closing time exceeded the bidders available time. However the clone in the second auction site continued as the payoff was better. 


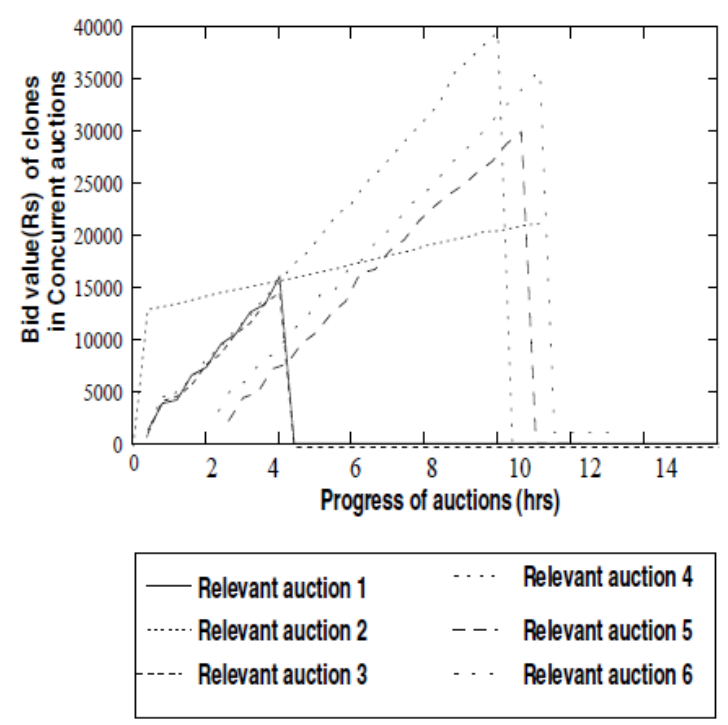

Fig. 2. Bid values Vs. progress of auctions

Decision making: The BDI agent is trained to participate in concurrent relevant auction sites and later to decide whether to continue bidding or withdraw from the auction. Various scenarios of auctions and types of co-bidders (risk neutral and averse) are considered for training. The BDI agent is trained for more than fifty scenarios of the bidding process and only a few of them are presented. The figures in 3, 4 and 5 shows the various scenarios considering the participation of few bidders along with the BDI agent for bidding and this agent is being trained to get attuned to various possible scenarios. In the aforesaid figures the number of times the BDI agent bids (calls) is represented on the $\mathrm{X}$-axis and the bid value on the Y-axis. The BDI agent creates clones of it and programs them to bid in auctions concurrently. The clones participates on-behalf of the master agent.

Scenario I (withdrawal situation) - Figure 3 depicts the BDI agent's decision to withdraw from bidding due to the aggressive attitude (measured in terms of bid increment value) shown by the co-bidders where the payoff of BDI reduces. The clone also predicted the closing price to be very high.

Scenario II (winning situation) - Figure 4 depicts that the bidding was aggressive initially but became linear as it progressed. Here BDI agent was also aggressive initially due to availability of the budget and continued to bid higher than the co-bidders at every call to bid of the auction as the payoff was higher. The figure depicts that the BDI agent has won after 27 th call to bid.
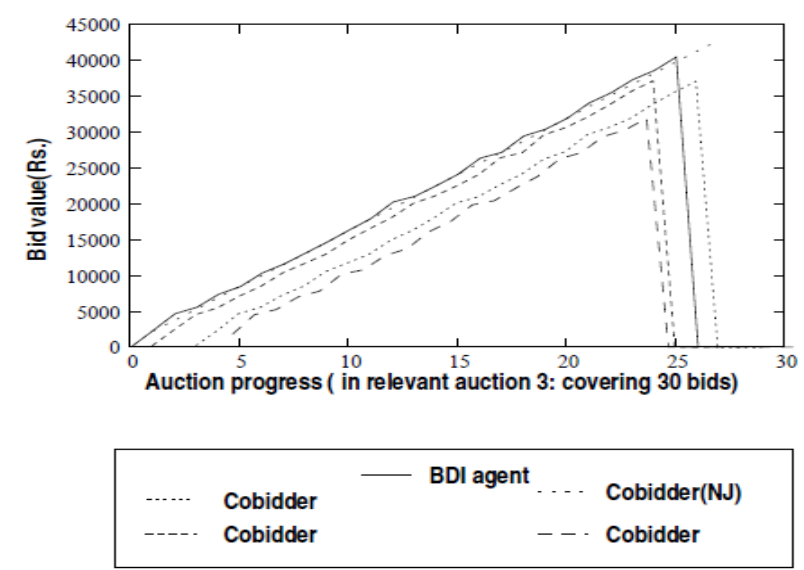

Fig. 3. Bid value Vs. bid calls of BDI agent (Scenario I)
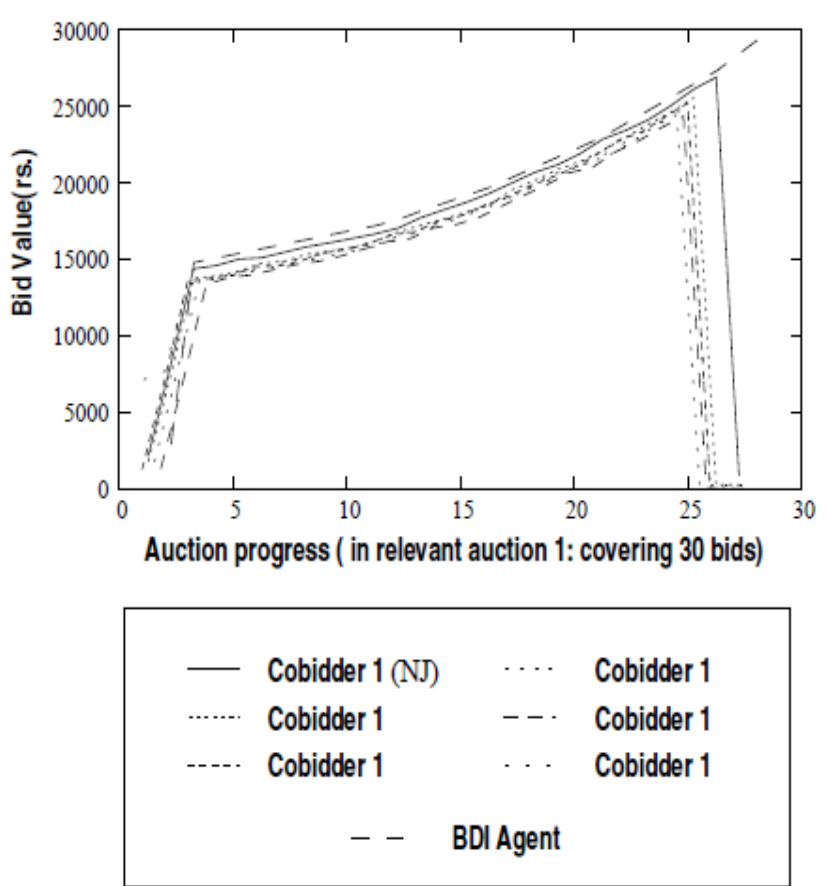

Fig. 4. Bid value Vs. bid calls of BDI agent (Scenario II)
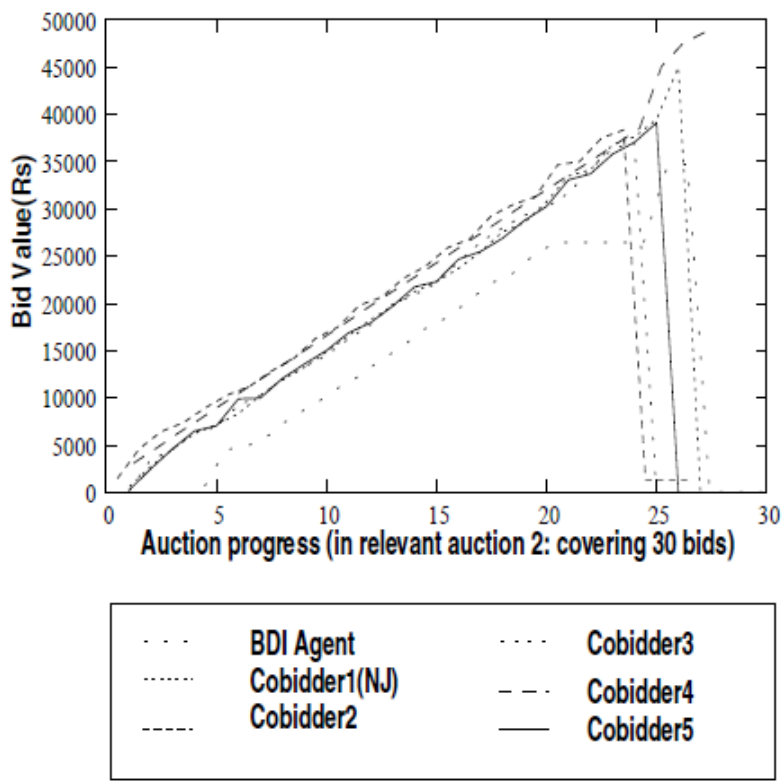

Fig. 5. Bid value Vs. bid calls of BDI agent (Scenario III)

Scenario III (withdrawal situation) - Figure 5 depicts that the BDI agent bids competitively with its co-bidders in every call to bid till it had sufficient budget. When the allotted budget to the BDI agent had to be reviewed the BDI slept(did not bid) from 20th call to 24th call and observed the trend of bidding of its co-bidders and continued to bid after its budget was reviewed and allotted. It withdrew itself when the co-bidders bid value was very high relatively and its payoff reduced.

After training the BDI agent for several scenarios. The BDI agent is ready to participate in bidding. Figures 6- 11 show the participation of the clones of the master agent along with the co-bidders. One of the co-bidder adopts NJ strategy to bid and the remaining co-bidders adopt random bidding strategy. 

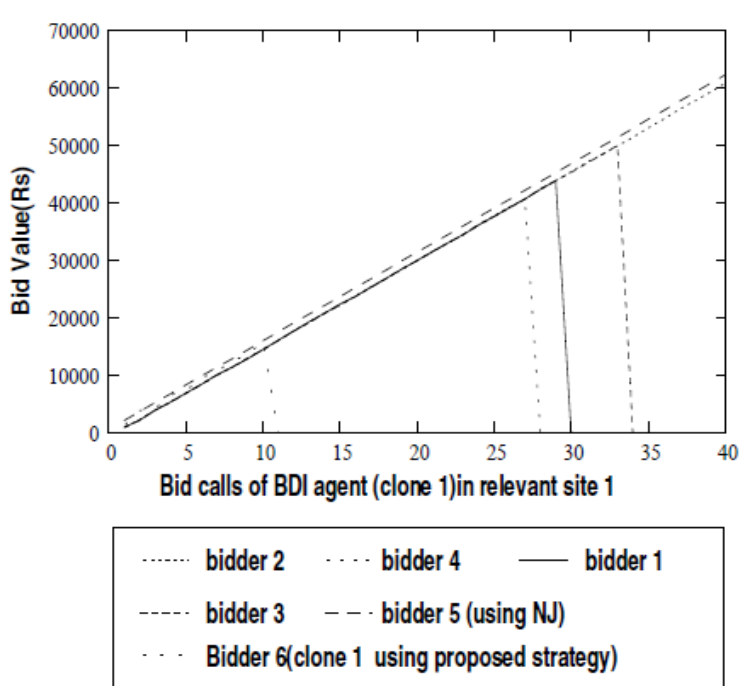

Fig. 6. BDI value Vs. bid calls of BDI agent (clone 1) in relevant site 4

Clone1 in figure 6 withdrew from bidding in relevant auction 4 as it was trained to withdraw if the rate of increase in bid value was higher than its rate of increase and budget was also reviewed once. The figure clearly showed that even after the clone withdrew the auction continued for many more iterations and concluded with a closing price much higher than the allotted and reviewed budget.

Clone 2 in relevant auction 2 as shown in figure 7 has bid competitively and won with a better price since the co-bidders were risk neutral. The clone has bid slightly aggressively initially since the co-bidders behavior were seen to be risk neutral. Once its bid values started becoming the maximum bid value among all bidders it maintained its bid rate till it won.

Clone 3 in figure 8 withdrew as soon as it predicted the closing price to be higher than its allotted budget and sensed that the bid value increment was higher and payoff was lesser compared to other concurrent auctions.

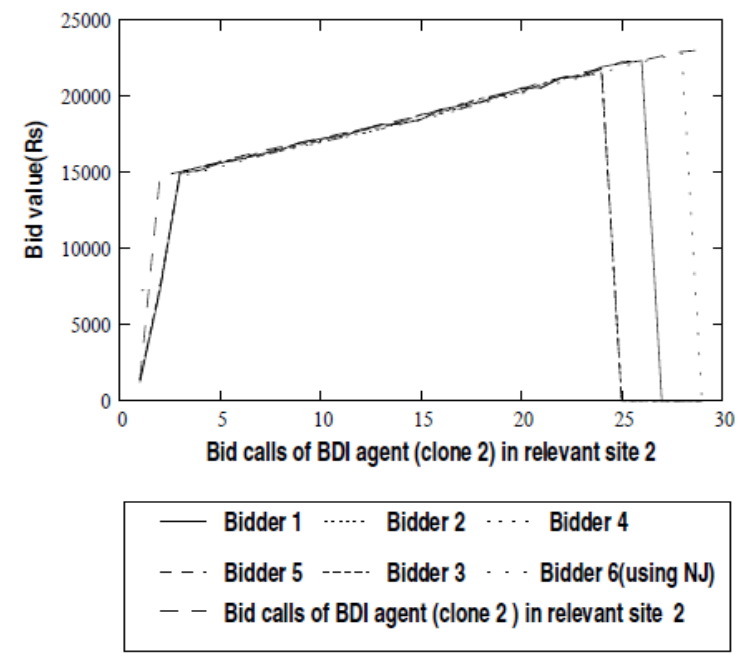

Fig. 7. BDI value Vs. bid calls of BDI agent (clone 2) in relevant site 2

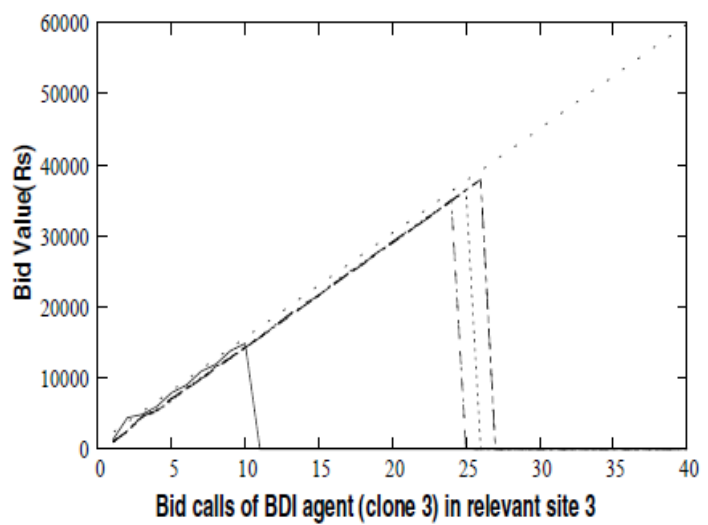

$$
\begin{aligned}
& \text {..... Bidder } 2 \text {---.. Bidder } 3 \text { - -. Bidder } 5 \\
& \text { Bidder } 4 \text { - Bidder } 1 \cdots \text { Bidder 6(using NJ) }
\end{aligned}
$$$$
\text { Bidder } 7 \text { (clone } 3 \text { using proposed strategy) }
$$

Fig. 8. BDI value Vs. bid calls of BDI agent (clone 3 ) in relevant site 3

Clone 4 in auction 4 as shown in figure 9 bid competitively with co-bidders and withdrew when its reviewed budget was exhausted.

Clone 5 in auction 5 as shown in figure 10 started bidding aggressively from the beginning and continued to bid highest value than co-bidders till its budget exhausted. It withdrew when it sensed that the co-bidders though not very aggressive in bidding but continued to bid for a longer time which naturally increased the bid value.

Clone 6 in figure 11 started bidding with a higher bid rate than others initially and then withdrew when there was a rise in bid rate of few co-bidders. As seen from the figure the bid ended at a price higher than the clones reviewed budget.

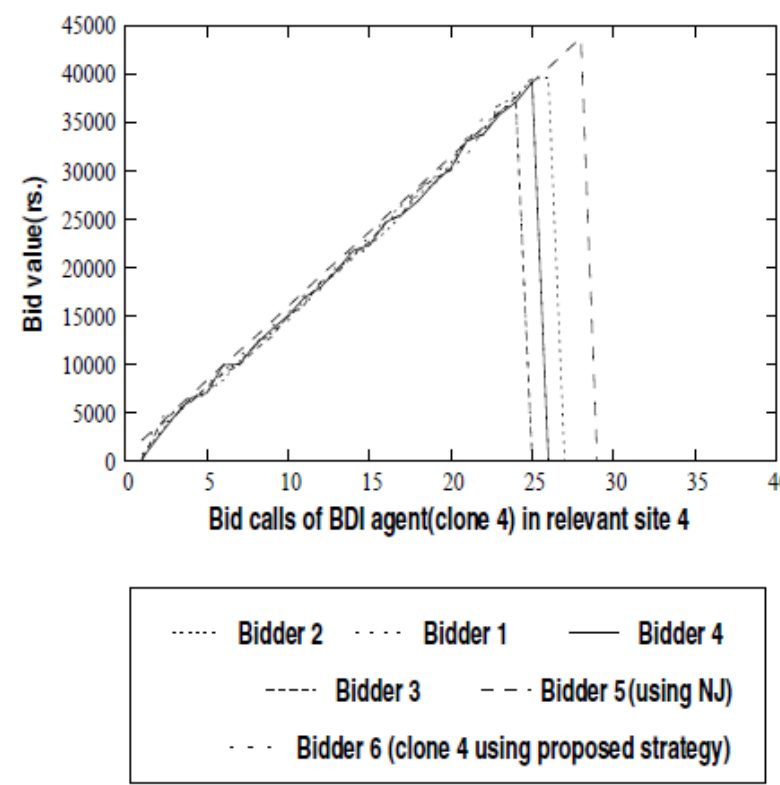

Fig. 9. BDI value Vs. bid calls of BDI agent (clone 4) in relevant site 4 

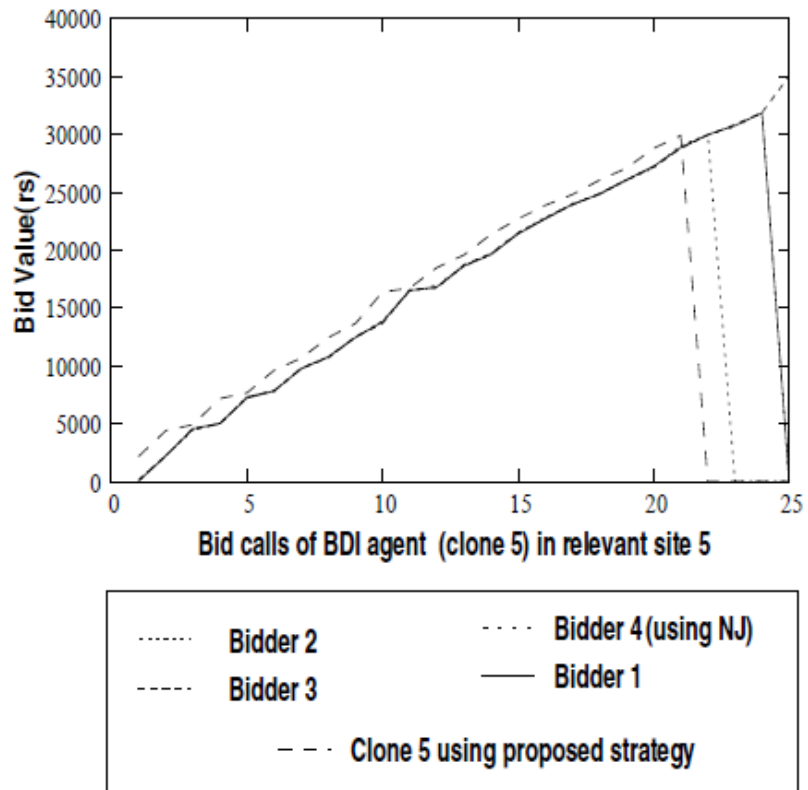

Fig. 10. BDI value Vs. bid calls of BDI agent (clone 5) in relevant site 5

Best possible winning bid (bid value): Figure 12 shows an instance of the bidding where the clones had participated in all the relevant auctions. The figures clearly showed that only one clone among all had won in a site that had better closing price. Rest of the clones was withdrawn from bidding in the concurrent relevant auctions. Clone 2 in figure 12 has bid aggressively initially and later linearly till it has won. The figure depicts that clone 2 has won the best possible bid among the concurrent relevant auctions.
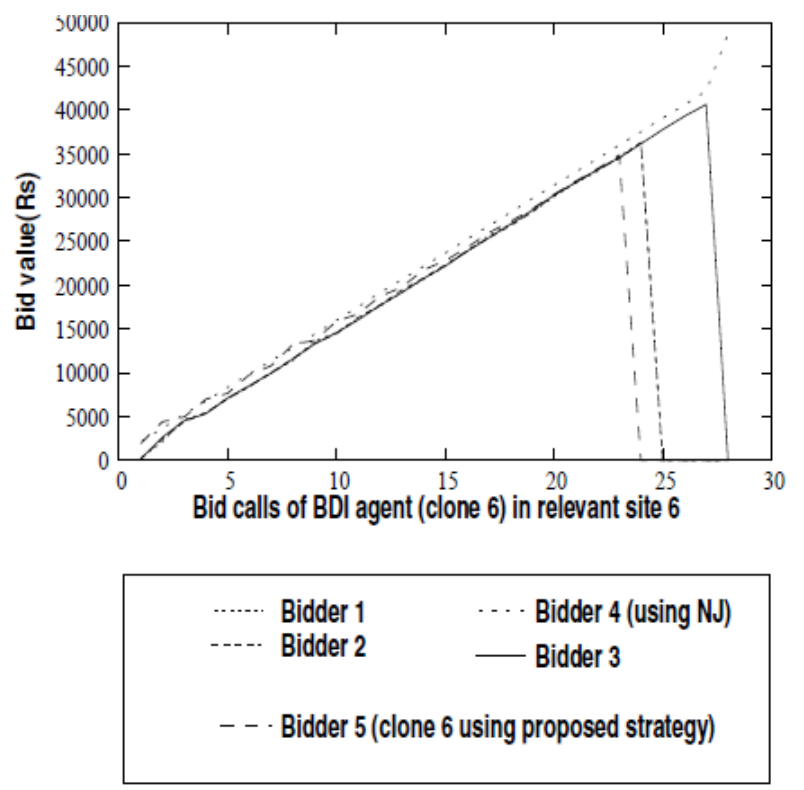

Fig. 11. BDI value Vs. bid calls of BDI agent (clone 6) in relevant site 6
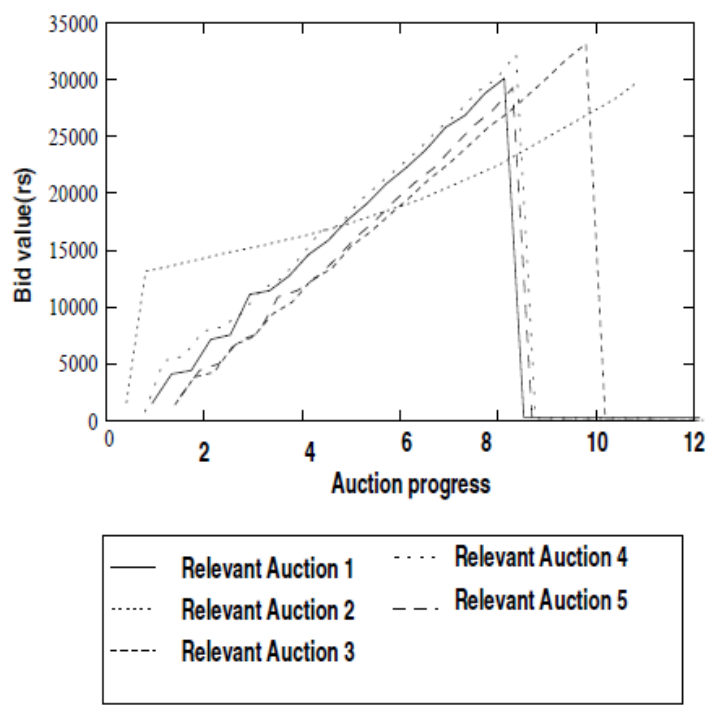

Fig. 12. Bid value Vs. auction progress

Bidders satisfaction - The figure in 13 show the satisfaction of the BDI agent that represents a bidder. It has variable satisfaction in the auctions due to the different probability to win, payoffs, predicted closing price within the allotted budget and absolute matching of the desired product with that of the available one in the relevant auctions.

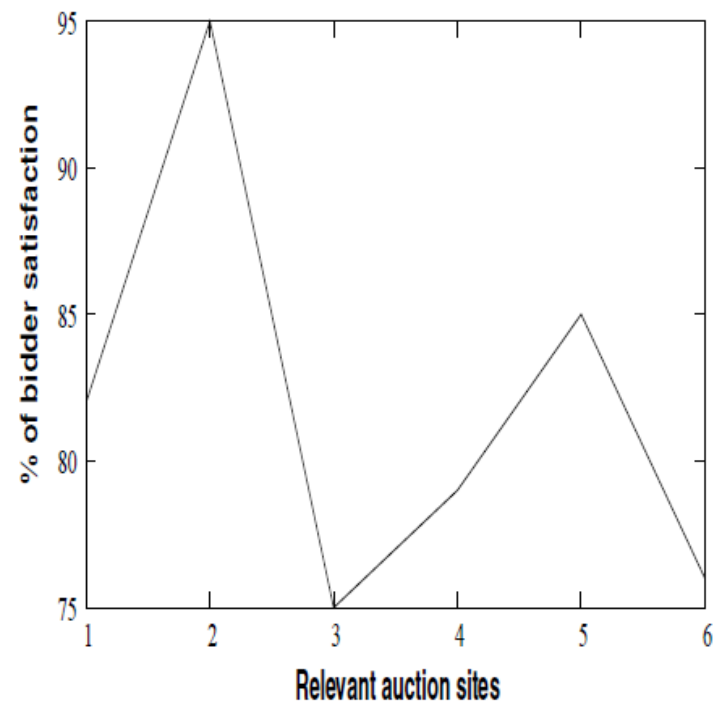

Fig. 13. \% Bidder satisfaction Vs. relevant auction sites

Initially budget is allotted to the master BDI agent and in turn it allots the same to the clones. Fig. 14 shows the remaining budget left with the participating clones after placing bid value in every round of the auction in all the relevant auctions. If the budget allotted to clones gets exhausted the master agent reviews the budget as shown for clone 4 in the Fig. 14. 


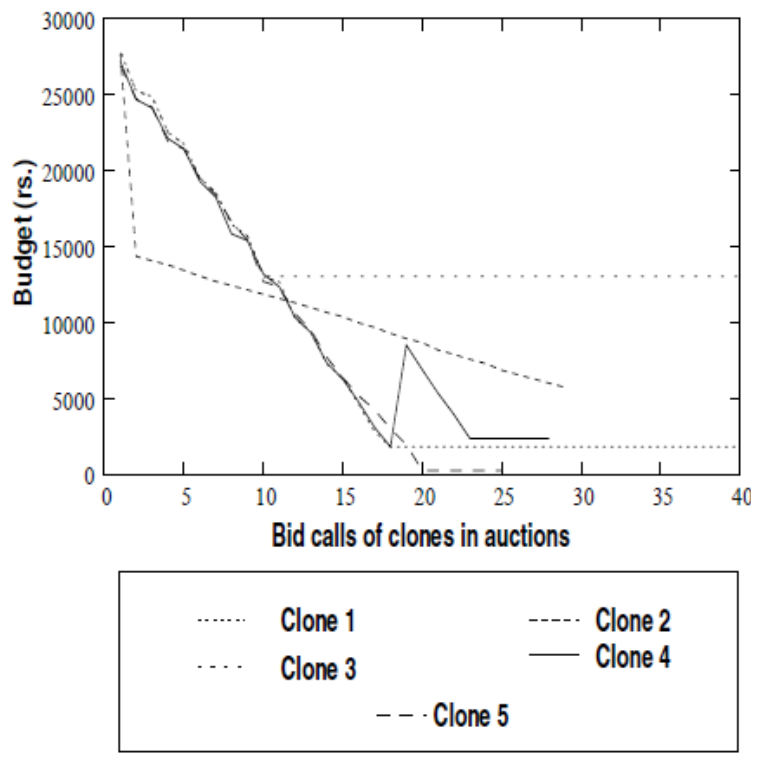

Fig. 14. Budget Vs. bid calls clones in auctions

\section{CONCLUSIONS}

This scheme proposes a scheme to design a framework to incorporate human intelligence and human perspective (cognitive) in agents for bidding in multiple concurrent English auctions in mobile E-commerce. The BDI agent is trained to carry out bidding in various scenarios of the e-auctions. The BDI agent is trained to compute bid value and bid along with the other bidders who may be risk averse or risk neutral while bidding. By doing so the BDI agent learns and gains experience to participate in auctions. During the training process the BDI agent learns to decide to bid, sleep or withdraw from the relevant sites. Learning is through simulation. This scheme may be generalized for all type of auctions.

\section{REFERENCES}

[1] Nandini S. Sidnal, Sunilkumar S. Manvi, "Are Mobile Agents Suitable for Mobile commerce?" in: Proc. of IEEE National Conference on Information and Communication Convergence (IEEE ICC 2006), Chennai, India, 2006, pp. 432-440.

[2] Anand R. Michael G., "Modeling rational agents within a BDI architecture," in: Proc. Second International Conference on Principles of Knowledge Representation and Reasoning, 1991, pp. 473-484

[3] Wolfgang Jank and Shu Zhang, "An Automated and Data- Driven Bidding Strategy for Online Auctions," INFORMS Journal on computing, vol 2, pp. 238-253, 2011.

[4] Minghua He, Nicholas R. Jennings, and Adam, "An Adaptive Bidding Agent for Multiple English Auctions: A Neuro-Fuzzy Approach." in: Proc. IEEE Conference on Fuzzy Systems, Budapest, Hungary, 2004, pp. 1519-1524.

[5] Patricia Anthony, Wendy Hall, Viet Dung Dang, and Nicholas R. Jennings, "Autonomous agents for participating in multiple online auctions," in Proc. Inter national joint conference on Artificial Intelligence workshop on E-Business and Intelligent Web, Seattle, 2001, pp 54-64.
[6] P. Chris, B. Claudio, P. Ivan, "Algorithm Design for Agents Which Participate in Multiple Simultaneous Auctions," in Proc. of Agent-Mediated Electronic Commerce III, Current issues in agents based electronic commerce systems, 2001, pp. 139-154.

[7] David C. K. Yuen, Andrew Byde, Nicholas R. Jennings, "Heuristic Bidding Strategies for Multiple Heterogeneous Auctions," in: Proc. 17th European Conference on Artificial Intelligence (ECAI 2006), Italy, 2006, pp 300-304.

[8] H. Minghua, J. Nicholas, P. Adam, "A heuristic bidding strategy for buying multiple goods in multiple English auctions," ACM Transactions on Internet Technology, vol. 4, pp. 465-496, 2006.

[9] Andrew Byde, Chris Preist, and Nicholas R. Jennings, "Decision Procedures for Multiple Auctions," in Proc. International Conference on Autonomous Agents - AAMAS(Agents), Italy, 2002, pp. 613-620.

[10] Marlon Dumas, Lachlan Aldred, and Guido Governatori, Arthur ter Hofstede, "Probabilistic Automated Bidding in Multiple Auctions," Journal of Electronic Commerce Research, vol. 1, 2005, pp. 23-47.

[11] Patrica Anthony and N. R. Jennings, "Developing a Bidding Agent for Multiple Heterogeneous Auctions," ACM Transactions on Internet Technology, vol 3, 2003, pp. 185-217.

[12] H. Minghua, J. Nicholas, P. Adam, "A heuristic bidding strategy for buying multiple goods in multiple english auctions," $A C M$ Transactions on Internet Technology, vol. 6, 2006, pp. 465-496.

[13] Nandini S. Sidnal and Sunilkumar S. Manvi, "Intelligent Agent Based Model for Auction Service Discovery in Mobile ECommerce," International Journal of Electronic Business Research, vol 1, 2011.

[14] Nandini S. Sidnal and Sunilkumar S. Manvi, "Cognitive Agent based Identification of Relevant Auctions in Mobile E-commerce," Journal of iBusiness, 2011, pp. 192-202, http://www.scirp.org/journal/ib.

[15] Nandini S. Sidnal and Sunilkumar S. Manvi, "BDI Agent based Final Price Prediction for English Auctions in Mobile E-Commerce," International Journal of World Review of Business Research (special issue), 2011.

Nandini Sidnal has completed her Ph. D and M.Tech. from Visvesvaraya Technological University. The title of her ph.d work was "Cognitive Agent based auction Service Discovery, Relevant Auction Identification and Bidding Mechanism", under the guidance of Dr. Sunil Kumar Manvi. She has completed her B.E in Computer Science and Engineering. She is currently working as Assistant Professor in Department of Computer Science and Engineering, K.L.E.S. College of Engineering and Technology, Belgaum, Karnataka. Her areas of interest include Distributed Computing, Mobile Agents, E-commerce, E-learning and Compiler Design, cl;oud computing. She has presented and published fifteen papers in referred National/ International Conferences. She is member of ISTE India and AgentLink UK

S. S. Manvi completed his $\mathrm{PhD}$, from Indian Institute of Science, Bangalore. Presently he is serving as a Dean Research and Development and Professor of Electronics and Communication Engineering, REVA Institute of Technology and Management, Bangalore. His areas of interest include wireless/wired network, AI applications in network management, Grid Computing and Multimedia Communications. He has published over 56 papers in referred National/International Journals and 108 papers in referred National/ International Conferences. $\mathrm{He}$ has coauthored books "Communication Protocol Engineering" and "Computer Concepts and C Programming" published by PHI. $\mathrm{He}$ is a reviewer of several national/international journals. He is a member of IEEE USA, Fellow of IETE, India Fellow of IE, India and member of ISTE, India. He has been included in Marqui's Who's who in world and International Biographies of Cambridge, London in the year 2006. Have been identified as one of the top 20 Indain scientists in the areas of Internet and Multimedia, Software Engg., and DBMS

This is as per the publication titled "Indian Computer Science Research Output During 1999-2008: Quantitave analysis" publsihed in DESIDOC Journal of Library and Information Technology, 2010. 